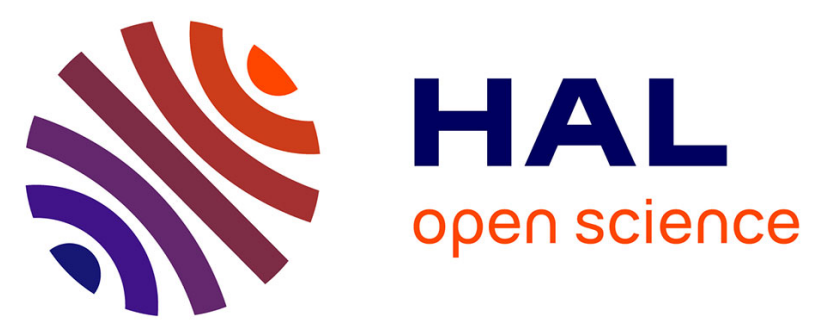

\title{
Robust absolute stability and nonlinear state feedback stabilization based on polynomial Lur'e functions
}

Vinícius F. Montagner, Ricardo C. L. F. Oliveira, T.R. Calliero, R. Borges, Pedro Luis Dias Peres, Christophe Prieur

\section{- To cite this version:}

Vinícius F. Montagner, Ricardo C. L. F. Oliveira, T.R. Calliero, R. Borges, Pedro Luis Dias Peres, et al.. Robust absolute stability and nonlinear state feedback stabilization based on polynomial Lur'e functions. Nonlinear Analysis: Theory, Methods and Applications, 2009, 70 (5), pp.1803-1812. hal00965332

\section{HAL Id: hal-00965332 \\ https://hal.science/hal-00965332}

Submitted on 27 Mar 2014

HAL is a multi-disciplinary open access archive for the deposit and dissemination of scientific research documents, whether they are published or not. The documents may come from teaching and research institutions in France or abroad, or from public or private research centers.
L'archive ouverte pluridisciplinaire HAL, est destinée au dépôt et à la diffusion de documents scientifiques de niveau recherche, publiés ou non, émanant des établissements d'enseignement et de recherche français ou étrangers, des laboratoires publics ou privés. 


\title{
Robust absolute stability and nonlinear state feedback stabilization based on polynomial Lur'e functions
}

\author{
$\begin{array}{lll}\text { V. F. Montagner } & \text { R. C. L. F. Oliveira } & \text { T. R. Calliero }\end{array}$ \\ $\begin{array}{ll}\text { R. A. Borges } & \text { P. L. D. Peres }\end{array}$ \\ C. Prieur $^{\S *}$ \\ * Power Electronics and Control Research Group \\ Federal University of Santa Maria \\ 97105-900, Santa Maria - RS - Brazil \\ $\dagger$ School of Electrical and Computer Engineering \\ University of Campinas \\ CP 6101, 13081-970, Campinas - SP - Brazil \\ $\S$ LAAS-CNRS, University of Toulouse, \\ 7, Avenue du Colonel Roche, 31077 Toulouse CEDEX 4 - France \\ E-mails: \\ montagne@smail.ufsm.br \\ \{ricfow,taisrc,renato,peres\}@dt.fee.unicamp.br \\ cprieur@laas.fr
}

\begin{abstract}
This paper provides finite dimensional convex conditions to construct homogeneous polynomially parameter-dependent Lur'e functions which ensure the stability of nonlinear systems with state-dependent nonlinearities lying in general sectors and which are affected by uncertain parameters belonging to the unit simplex. The proposed conditions are written as linear matrix inequalities parametrized in terms of the degree $g$ of the parameterdependent solution and in terms of the relaxation level $d$ of the inequality constraints, based on algebraic properties of positive matrix polynomials with parameters in the unit simplex. As $g$ and $d$ increase, progressively less conservative solutions are obtained. The results in the paper include as special cases existing conditions for robust stability and for absolute stability analysis. A convex solution suitable for the design of robust nonlinear state feedback stabilizing controllers is also provided. Numerical examples illustrate the efficiency of the proposed conditions.
\end{abstract}

${ }^{*}$ Corresponding author. 


\section{Introduction}

The stability of dynamic systems affected by sector nonlinearities is an important issue in system theory, with application in several problems as in the study of neural networks, ecosystems, saturated nonlinear systems, systems with delays, among others [1,5,16-18]. The approach based on Lyapunov functions can be used to investigate the stability of this class of systems, providing tests in the form of linear matrix inequalities (LMIs) that are attractive due to the fact that the problem can be solved by means of polynomial time algorithms with global convergence $[8,12,29]$. For instance, the stability of nonlinear systems with state dependent nonlinearities belonging to infinite sectors can be investigated using an integral Lyapunov function (called Persidskii function [23]), implying that the existence of a diagonal positive definite matrix solving the Lyapunov inequality is sufficient to ensure stability for all nonlinear functions in the sector. For this class of nonlinear systems, the $S$-procedure and quadratic Lyapunov functions can be used to verify the absolute stability, leading to the well known circle condition [8]. Lyapunov functions that are quadratic on the state and integral on the nonlinearities (called Lur'e functions) lead to the Popov criterion, with a frequency domain interpretation related to the strict positive realness of a transfer function $[8,24,30]$. In the context of control synthesis, the circle and the Popov criteria have been recently investigated in [2,3] to derive conditions for nonlinear feedback design based on LMIs.

However, when dealing with sector nonlinearities, most of approaches do not take into account that all the system matrices can be affected by uncertain parameters. In this case, approaches based on a parameter-independent matrix in the Lyapunov function can lead to conservative results. It is known in the context of robust stability of linear systems affected by uncertainties that the conservatism of the results based on Lyapunov functions reduces when one uses affine parameter-dependent Lyapunov functions [11, 13, 22, 25] when compared to parameter-independent Lyapunov functions [4, 8]. More recently, it has been shown that if there exists a solution for a parameter-dependent LMI with parameters in a compact set then, without loss of generality, there exists a polynomially parameter-dependent solution [6]. Conditions to construct such solutions have been given in $[9,15,21,28]$. In this context, the study of absolute stability of uncertain systems based on polynomially parameter-dependent Lyapunov functions is a matter that deserves deeper investigation.

The main objective of this paper is the analysis of stability of systems for which all matrices depend on uncertain time-invariant parameters lying in the unit simplex and that are affected by state-dependent nonlinearities belonging to a general class of sectors. The proposed conditions rely on the feasibility of parameter-dependent LMIs whose solution provides a parameter-dependent Lur'e function which ensures stability for the entire domain of uncertainties and for all nonlinearities belonging to the sector. It is shown that, without loss of generality, the solution of such parameter-dependent LMIs is given by homogeneous polyno- 
mially parameter-dependent (HPPD) matrices. Then, necessary and sufficient LMI conditions to construct these HPPD matrices with arbitrary degree are given, using progressively less conservative LMI relaxations provided by an extension of Pólya's Theorem [14] to the case of matrix-valued polynomials [20,26,27]. The proposed conditions include as special cases the results in [10], concerned with absolute stability of precisely known systems, and the results in [21], for robust stability of uncertain polytopic systems. A convex condition for synthesis of robust stabilizing controllers based on the feedback of linear and nonlinear functions of the state is also provided, being the results in this case also valid for arbitrary time-varying parameters. Numerical examples illustrate the efficiency of the conditions given in the paper.

\section{Problem formulation}

Consider the system

$$
\dot{x}=A(\alpha) x+\tilde{A}(\alpha) f(x)+B(\alpha) u
$$

where $x \in \mathbb{R}^{n}$ is the state, $u \in \mathbb{R}^{m}$ is the control input, matrices $A(\alpha) \in \mathbb{R}^{n \times n}, \tilde{A}(\alpha) \in \mathbb{R}^{n \times n}$ and $B(\alpha) \in \mathbb{R}^{n \times m}$ lie inside the polytope

$$
\mathscr{P}=\left\{(A, \tilde{A}, B)(\alpha):(A, \tilde{A}, B)(\alpha)=\sum_{i=1}^{N} \alpha_{i}(A, \tilde{A}, B)_{i}, \alpha \in \mathscr{U}\right\}
$$

with given vertices $A_{i}, \tilde{A}_{i}, B_{i}, i=1, \ldots, N$, where $\alpha \in \mathbb{R}^{N}$ is the vector of uncertain timeinvariant parameters belonging to the unit simplex

$$
\mathscr{U}=\left\{\alpha \in \mathbb{R}^{N}: \sum_{i=1}^{N} \alpha_{i}=1, \alpha_{i} \geq 0, i=1, \ldots, N\right\}
$$

Vector $f(x)$ is a vector of nonlinear functions of the state, which are radially unbounded, fulfilling the general class of sector functions given by $[8,10]$

$$
\begin{aligned}
& \mathscr{F}(\gamma, \delta)=\{x \rightarrow f(x)= {\left[f_{1}\left(x_{1}\right) \ldots f_{n}\left(x_{n}\right)\right]^{\prime}: } \\
&\left.\gamma_{i} \tau^{2} \leq f_{i}(\tau) \tau \leq \delta_{i} \tau^{2}, 0<\gamma_{i} \leq 1 \leq \delta_{i}, \quad f_{i}(0)=0, i=1, \ldots, n\right\}
\end{aligned}
$$

Notice that $\mathscr{F}(\gamma, \delta)$ can cover an infinite sector by choosing $\gamma_{i}=\beta, \delta_{i}=1 / \beta$ and $\lim \beta \rightarrow 0^{+}$.

The objective of this paper is to address the following problems.

Problem 1 Determine if the nonlinear system (1), with $u=\mathbf{0}$, is globally asymptotically stable $(G A S)$ to $x=\mathbf{0}, \forall f \in \mathscr{F}(\gamma, \delta), \forall \alpha \in \mathscr{U}$. In other words, determine if the autonomous system is robustly (with respect to $\alpha$ ) absolutely (with respect to $f(x)$ ) GAS to $x=\mathbf{0}$.

Problem 2 Assume that $x$ and $f(x)$ are available for feedback through the control law

$$
u=K x+\tilde{K} f(x)
$$


Determine $K \in \mathbb{R}^{m \times n}$ and $\tilde{K} \in \mathbb{R}^{m \times n}$ such that the closed-loop system

$$
\dot{x}=A_{c l}(\alpha) x+\tilde{A}_{c l}(\alpha) f(x), A_{c l}(\alpha)=A(\alpha)+B(\alpha) K, \tilde{A}_{c l}(\alpha)=\tilde{A}(\alpha)+B(\alpha) \tilde{K}
$$

is $G A S$ to $x=\mathbf{0}, \forall f \in \mathscr{F}(\gamma, \delta), \forall \alpha \in \mathscr{U}$.

\section{Preliminary results}

Theorem 1 Consider a parameter-dependent LMI generally written as

$$
G(\xi, \alpha)=G_{0}(\alpha)+\xi_{1} G_{1}(\alpha)+\ldots+\xi_{M} G_{M}(\alpha)>0
$$

where $\alpha \in \mathscr{U}$. Assume that $G_{i}(\alpha), i=0, \ldots, M$ are continuous functions. If $\forall \alpha \in \mathscr{U}$ there exists a parameter-dependent solution $\xi(\alpha) \in \mathbb{R}^{M}$ such that $G(\xi(\alpha), \alpha)>0$, then there exists an HPPD solution $\xi^{*}(\alpha): \mathscr{U} \rightarrow \mathbb{R}^{M}$ such that, $\forall \alpha \in \mathscr{U}, G\left(\xi^{*}(\alpha), \alpha\right)>0$.

Proof: In [6], it has been shown that, with the assumptions of Theorem 1, if there exists a solution $\xi(\alpha) \in \mathbb{R}^{M}$ for the parameter-dependent LMI (7), then there exists, with no loss of generality, a polynomial solution $\xi^{* *}(\alpha)$ such that, $\forall \alpha \in \mathscr{U}, G\left(\xi^{* *}(\alpha), \alpha\right)>0$. Assuming that $g$ is the largest degree present in the monomials of $\xi^{* *}(\alpha)$, one can write

$$
\xi^{* *}(\alpha)=\sum_{0 \leq \beta_{1}+\cdots+\beta_{N} \leq g, \beta_{i} \geq 0} c_{\beta_{1}, \ldots, \beta_{N}} \alpha_{1}^{\beta_{1}} \ldots \alpha_{N}^{\beta_{N}}
$$

Consider now the following homogeneous polynomial $\xi^{*}(\alpha)$ of degree $g$ :

$$
\xi^{*}(\alpha)=\sum_{0 \leq \beta_{1}+\cdots+\beta_{N} \leq g, \beta_{i} \geq 0} c_{\beta_{1}, \ldots, \beta_{N}} \alpha_{1}^{\beta_{1}} \ldots \alpha_{N}^{\beta_{N}}\left(\sum_{i=1}^{N} \alpha_{i}\right)^{g-\sum_{i} \beta_{i}}
$$

Clearly, $\xi^{*}(\alpha)$ coincides with $\xi^{* *}(\alpha)$ in $\mathscr{U}$. Thus, the homogeneous polynomial $\xi^{*}(\alpha)$ is a solution for the parameter-dependent LMI (7) for any value of $\alpha \in \mathscr{U}$.

More details on the result presented in Theorem 1 can be seen in [7].

By combining Theorem 1 and the results in [10], a sufficient condition to solve Problem 1 is expressed in the next theorem.

Theorem 2 For given diagonal matrices $\Gamma=\operatorname{diag}\left\{\gamma_{i}\right\}, \Delta=\operatorname{diag}\left\{\delta_{i}\right\}$, with $0<\gamma_{i} \leq 1 \leq \delta_{i}$, $i=1, \ldots, n$, if $\forall \alpha \in \mathscr{U}$, there exist diagonal positive definite HPPD matrices $P_{g}(\alpha) \in \mathbb{R}^{n \times n}$, $Q_{g}(\alpha) \in \mathbb{R}^{n \times n}$ and a symmetric HPPD matrix $W_{g}(\alpha) \in \mathbb{R}^{n \times n}$ such that (the symbol $\star$ denotes symmetric blocks in the LMIs)

$$
\begin{gathered}
W_{g}(\alpha)+P_{g}(\alpha) \Gamma>0 \\
{\left[\begin{array}{cc}
\mathscr{T}_{11}(\alpha) & \mathscr{T}_{12}(\alpha) \\
\star & \mathscr{T}_{22}(\alpha)
\end{array}\right]<0}
\end{gathered}
$$


with

$$
\begin{gathered}
\mathscr{T}_{11}(\alpha) \triangleq \tilde{A}(\alpha)^{\prime} P_{g}(\alpha)+P_{g}(\alpha) \tilde{A}(\alpha)-Q_{g}(\alpha) \\
\mathscr{T}_{12}(\alpha) \triangleq \tilde{A}(\alpha)^{\prime} W_{g}(\alpha)+P_{g}(\alpha) A(\alpha)+\frac{1}{2}(\Gamma+\Delta) Q_{g}(\alpha) \\
\mathscr{T}_{22}(\alpha) \triangleq A(\alpha)^{\prime} W_{g}(\alpha)+W_{g}(\alpha) A(\alpha)-\Gamma \Delta Q_{g}(\alpha)
\end{gathered}
$$

then system (1), with $u=\mathbf{0}$, is GAS to $x=\mathbf{0}, \forall f \in \mathscr{F}(\gamma, \delta), \forall \alpha \in \mathscr{U}$.

Proof: Consider the parameter-dependent Lur'e function

$$
v(x)=x^{\prime} W(\alpha) x+2 \sum_{i=1}^{n} p_{i i}(\alpha) \int_{0}^{x_{i}} f_{i}(\tau) d \tau
$$

as a Lyapunov candidate function. From (4), one has that $\int_{0}^{x_{i}} \gamma_{i} \tau d \tau \leq \int_{0}^{x_{i}} f_{i}(\tau) d \tau$, implying that the existence of a symmetric matrix $W(\alpha)$ and a diagonal positive definite $P(\alpha)$ such that

$$
W(\alpha)+P(\alpha) \Gamma>0, \forall \alpha \in \mathscr{U}
$$

with $\Gamma=\operatorname{diag}\left\{\gamma_{i}\right\}$, is sufficient to ensure $v(x)>0, \forall \alpha \in \mathscr{U}, \forall x \neq \mathbf{0}$. The time-derivative of (10) along the trajectories of system (1) with $u=\mathbf{0}$ results in

$$
\dot{v}(x)=\eta^{\prime}\left[\begin{array}{cc}
\mathscr{S}_{11}(\alpha) & \mathscr{S}_{12}(\alpha) \\
\star & \mathscr{S}_{22}(\alpha)
\end{array}\right] \eta
$$

with $\eta^{\prime}=\left[\begin{array}{ll}f(x)^{\prime} & x^{\prime}\end{array}\right]$ and

$$
\begin{aligned}
& S_{11}(\alpha) \triangleq \tilde{A}(\alpha)^{\prime} P(\alpha)+P(\alpha) \tilde{A}(\alpha) \\
& \mathscr{S}_{12}(\alpha) \triangleq \tilde{A}(\alpha)^{\prime} W(\alpha)+P(\alpha) A(\alpha) \\
& \mathscr{S}_{22}(\alpha) \triangleq A(\alpha)^{\prime} W(\alpha)+W(\alpha) A(\alpha)
\end{aligned}
$$

For all $f(x) \in \mathscr{F}(\gamma, \delta)$, one has that

$$
\sum_{i=1}^{n}\left(f_{i}\left(x_{i}\right)-\gamma_{i} x_{i}\right) q_{i i}(\alpha)\left(f_{i}\left(x_{i}\right)-\delta_{i} x_{i}\right) \leq 0
$$

is valid $\forall q_{i i}(\alpha)>0, \forall \alpha \in \mathscr{U}$. Thus,

$$
\Theta \triangleq \eta^{\prime}\left[\begin{array}{cc}
Q(\alpha) & -\frac{1}{2}(\Gamma+\Delta) Q(\alpha) \\
\star & \Gamma \Delta Q(\alpha)
\end{array}\right] \eta \leq 0
$$

for any $Q(\alpha)=\operatorname{diag}\left\{q_{i i}(\alpha)\right\}$, for $\Gamma=\operatorname{diag}\left\{\gamma_{i}\right\}$ and $\Delta=\operatorname{diag}\left\{\delta_{i}\right\}$, with $0<\gamma_{i} \leq 1 \leq \delta_{i}$. Since $\dot{v}(x) \leq \dot{v}(x)-\Theta<0$, one has that, $\forall \eta \neq \mathbf{0}$, the existence of $W(\alpha)$ symmetric, and $P(\alpha)$ and $Q(\alpha)$ diagonal positive definite such that

$$
\left[\begin{array}{cc}
\mathscr{S}_{11}(\alpha)-Q(\alpha) & \mathscr{S}_{12}(\alpha)+\frac{1}{2}(\Gamma+\Delta) Q(\alpha) \\
\star & \mathscr{S}_{22}(\alpha)-\Gamma \Delta Q(\alpha)
\end{array}\right]<0
$$


$\forall \alpha \in \mathscr{U}$ ensures that $\dot{v}(x)<0 \forall \alpha \in \mathscr{U}, \forall f \in \mathscr{F}(\gamma, \delta)$. Thus, if the parameter-dependent LMIs (11) and (12) have a solution given by $P(\alpha), Q(\alpha)$ and $W(\alpha)$, then $v(x)$ in (10) is a Lur'e function ensuring robust absolute stability for the system. Finally, from Theorem 1, it follows that this conclusion holds for HPPD matrices $P_{g}(\alpha), Q_{g}(\alpha)$ and $W_{g}(\alpha)$, which allows to state Theorem 2 in terms of the search of HPPD matrices, with no loss of generality.

In the sequel, some notation and definitions are introduced in order to construct HPPD matrices of arbitrary degree that solve Theorem 2 .

\section{Notation and definitions}

A homogeneous matrix-polynomial $P_{g}(\alpha)$ of degree $g$ can be generally written as

$$
P_{g}(\alpha)=\sum_{k \in \mathscr{K}(g)} \alpha_{1}^{k_{1}} \alpha_{2}^{k_{2}} \cdots \alpha_{N}^{k_{N}} P_{k}, k=k_{1} k_{2} \cdots k_{N}
$$

where $\alpha_{1}^{k_{1}} \alpha_{2}^{k_{2}} \cdots \alpha_{N}^{k_{N}}, \alpha \in \mathscr{U}, k_{i} \in \mathbb{Z}_{+}$(nonnegative integers), $i=1, \ldots, N$ are the monomials, and $P_{k} \in \mathbb{R}^{n \times n}, \forall k \in \mathscr{K}(g)$ are matrix-valued coefficients. Here, by definition, $\mathscr{K}(g)$ is the set of $N$-tuples obtained as all possible combinations of nonnegative integers $k_{i}, i=1, \ldots, N$, such that $k_{1}+k_{2}+\ldots+k_{N}=g$. Since the number of vertices in the polytope $\mathscr{P}$ is equal to $N$, the number of elements in $\mathscr{K}(g)$ is given by ${ }^{1}$

$$
J(g)=\frac{(N+g-1) !}{g !(N-1) !}
$$

To give an example, for homogeneous polynomials of degree $g=3$ with $N=2$ variables, the possible values of the partial degrees are $\mathscr{K}(3)=\{03,12,21,30\}$ (so $J(3)=4$ ), corresponding to the generic form $P_{3}(\alpha)=\alpha_{2}^{3} P_{03}+\alpha_{1} \alpha_{2}^{2} P_{12}+\alpha_{1}^{2} \alpha_{2} P_{21}+\alpha_{1}^{3} P_{30}$. Constant (zero-degree) matrices are obtained from (13) for $g=0$.

By definition, for $N$-tuples $k, k^{\prime}$ one writes $k \succeq k^{\prime}$ if $k_{i} \geq k_{i}^{\prime}, i=1, \ldots, N$. Usual operations of summation $k+k^{\prime}$ and subtraction $k-k^{\prime}$ (whenever $k \succeq k^{\prime}$ ) are defined componentwise. Consider also the following definitions for the $N$-tuple $e_{i}$ and the coefficient $\pi(k)$

$$
e_{i}=0 \cdots 0 \underbrace{1}_{i-t h} 0 \cdots 0, \quad \pi(k) \triangleq\left(k_{1} !\right)\left(k_{2} !\right) \cdots\left(k_{N} !\right)
$$

\section{Main results}

Next theorem provides a necessary and sufficient finite dimensional convex condition to solve Theorem 2.

Theorem 3 Given diagonal matrices $\Gamma=\operatorname{diag}\left\{\gamma_{i}\right\}, \Delta=\operatorname{diag}\left\{\delta_{i}\right\}$, with $0<\gamma_{i} \leq 1 \leq \delta_{i}, i=$ $1, \ldots, n$, there exist HPPD matrices of arbitrary degree $g$ solving Theorem 2 if, and only if, there

\footnotetext{
${ }^{1}$ The symbol (!) denotes factorial.
} 
exist diagonal matrices $P_{k} \in \mathbb{R}^{n \times n}, Q_{k} \in \mathbb{R}^{n \times n}$, symmetric matrices $W_{k} \in \mathbb{R}^{n \times n}, k \in \mathscr{K}(\mathrm{g})$, and a sufficiently large $d \in \mathbb{Z}_{+}$such that the following LMIs hold

$$
\begin{gathered}
\mathscr{N}_{1_{k}} \triangleq \sum_{\substack{k^{\prime} \in \mathscr{K}(d) \\
k \succeq k^{\prime}}} \frac{d !}{\pi\left(k^{\prime}\right)}\left(P_{k-k^{\prime}}\right)>0, \quad \forall k \in \mathscr{K}(g+d) \\
\mathscr{N}_{2_{k}} \triangleq \sum_{\substack{k^{\prime} \in \mathscr{K}(d) \\
k \succeq k^{\prime}}} \frac{d !}{\pi\left(k^{\prime}\right)}\left(Q_{k-k^{\prime}}\right)>0, \quad \forall k \in \mathscr{K}(g+d) \\
\mathscr{M}_{1_{k}} \triangleq \sum_{\substack{k^{\prime} \in \mathscr{K}(d) \\
k \succeq k^{\prime}}} \sum_{\substack{i \in\{1, \ldots, N\} \\
k_{i}>k_{i}^{\prime}}} \frac{d !}{\pi\left(k^{\prime}\right)}\left(W_{k-k^{\prime}-e_{i}}+\Gamma P_{k-k^{\prime}-e_{i}}\right)>0, \quad \forall k \in \mathscr{K}(g+d+1) \\
\mathscr{M}_{2_{k}} \triangleq \sum_{\substack{k^{\prime} \in \mathscr{K}(d) \\
k \succeq k^{\prime}}} \sum_{\substack{i \in\{1, \ldots, N\} \\
k_{i}>k_{i}^{\prime}}} \frac{d !}{\pi\left(k^{\prime}\right)}\left[\begin{array}{cc}
\mathscr{X}_{11} & \mathscr{X}_{12} \\
\star & \mathscr{X}_{22}
\end{array}\right]<0, \quad \forall k \in \mathscr{K}(g+d+1)
\end{gathered}
$$

with

$$
\begin{gathered}
\mathscr{X}_{11} \triangleq \tilde{A}_{i}^{\prime} P_{k-k^{\prime}-e_{i}}+P_{k-k^{\prime}-e_{i}} \tilde{A}_{i}-Q_{k-k^{\prime}-e_{i}}, \quad \mathscr{X}_{12} \triangleq \tilde{A}_{i}^{\prime} W_{k-k^{\prime}-e_{i}}+P_{k-k^{\prime}-e_{i}} A_{i}+\frac{1}{2}(\Gamma+\Delta) Q_{k-k^{\prime}-e_{i}} \\
\mathscr{X}_{22} \triangleq A_{i}^{\prime} W_{k-k^{\prime}-e_{i}}+W_{k-k^{\prime}-e_{i}} A_{i}-\Gamma \Delta Q_{k-k^{\prime}-e_{i}}
\end{gathered}
$$

Proof: If there exists, for a given degree $g$, a symmetric HPPD matrix $P_{g}(\alpha)>0$ for all $\alpha \in \mathscr{U}$, then for any $d \in \mathbb{Z}_{+}$

$$
\left(\sum_{i=1}^{N} \alpha_{i}\right)^{d} P_{g}(\alpha)=\sum_{k \in \mathscr{K}(g+d)} \alpha_{1}^{k_{1}} \alpha_{2}^{k_{2}} \ldots \alpha_{N}^{k_{N}} \mathscr{N}_{1_{k}}, \quad k=k_{1} k_{2} \ldots k_{N}
$$

with $\mathscr{N}_{1_{k}}$ given by (14) is an HPPD matrix of degree $g+d$ that is positive definite. Using an extension of Pólya's Theorem to the case of matrix valued polynomials [20, 26, 27], there exists a sufficiently large $d \in \mathbb{Z}_{+}$such that (18) has all the terms $\mathscr{N}_{1_{k}}>0, k \in \mathscr{K}(g+d)$. Conversely, it is clear that if $\mathscr{N}_{1_{k}}>0$, then $P_{g}(\alpha)>0 \forall \alpha \in \mathscr{U}$. The same is applicable to demonstrate that (15), (16) and (17) are sufficient and necessary (for sufficiently large $d \in \mathbb{Z}_{+}$) to ensure that $Q_{g}(\alpha)>0$, and that (8) and (9) are feasible $\forall \alpha \in \mathscr{U}$.

As a first remark on Theorem 3, one has that the increase on $d$ allows to reduce the conservatism of the results due to relaxations of the LMIs (with no increase in the number of decision variables for a given degree $g$ ). Moreover, if the LMIs of Theorem 3 are feasible for $\hat{d} \in \mathbb{Z}_{+}$, then these LMIs are feasible for any $d>\hat{d}$ since the LMIs for $\hat{d}+1$ can be written as positive combinations of the LMIs for $\hat{d}$. The use of higher $g$ for the matrix variables of Theorem 3 introduce more scalar variables in the problem, which can also reduce the conservatism of the results 
(see [19]). The number of scalar variables to solve Theorem 3 is $\mathscr{V}=(2 n+n(n+1) / 2) J(g)$ and the number of LMI rows is $\mathscr{R}=2 n J(g+d)+3 n J(g+d+1)$. Using interior point based algorithms as the LMI Control Toolbox of Matlab [12], this problem can be solved in polynomial time (proportional to $\mathscr{V}^{3} \mathscr{R}$ ).

The conditions of Theorem 3 can be specialized to robust stability analysis of linear systems by setting $\tilde{A}_{i}$ and the matrix coefficients $P_{k}$ to zero. In this case, condition (16) ensures that $W_{g}(\alpha)>0$ and condition (17), with the choice $\Gamma=\Delta=\mathbf{I}$, becomes necessary and sufficient to solve $A(\alpha)^{\prime} W_{g}(\alpha)+W_{g}(\alpha) A(\alpha)<0 \forall \alpha \in \mathscr{U}$, recovering the results from [19] for robust stability analysis.

Theorem 3 can be used to evaluate robust absolute stability of system (1) with $A(\alpha)=\mathbf{0}$ and $u=\mathbf{0}$. Setting matrices $A_{i}$ to zero and $\tilde{A}_{i}=A$ in Theorem 3, and choosing $g=0$ (i.e. fixed matrix variables), the conditions of Theorem 3 reduce to the conditions from [10]. However, the use of parameter-independent matrices in Theorem 3 to cope with the stability analysis for uncertain matrices $\tilde{A}(\alpha)$ can lead to conservative results. On the other hand, progressively less conservative results can be obtained by increasing $g$ and $d$, thanks to the general formulation of the conditions of Theorem 3.

Suppose (14)-(17) are feasible for $A_{i}=\mathbf{0}$, providing as a solution matrices to construct $P_{g}(\alpha), Q_{g}(\alpha)$ and $W_{g}(\alpha)$. The frequency domain interpretation given in [10] for the case of the system with precisely known matrices is directly applicable to the case of matrices affected by uncertainties studied here. For instance, the feasibility of Theorem 3 is equivalent to the fact that the transfer function

$$
M(s, \alpha)=\left(\frac{1}{2} Q_{g}(\alpha)(\Gamma-\Delta)-P_{g}(\alpha) \tilde{A}(\alpha) \Gamma\right)(s \mathbf{I}-\tilde{A}(\alpha))^{-1} \tilde{A}(\alpha)+\left(\frac{1}{2} Q_{g}(\alpha)-P_{g}(\alpha) \tilde{A}(\alpha)\right)
$$

is strictly positive real (see [10, Theorem 3]). Moreover, following [10, Lemma 9], if the circle or the Popov criteria are satisfied, then Theorem 3 will be feasible.

It is also interesting to mention that the evaluation of Theorem 3 with $A_{i}=\mathbf{0}$ and the matrix coefficients $W_{k}=\mathbf{0}$ leads to the analysis of stability of the autonomous uncertain nonlinear system (1) by means of a parameter-dependent Lur'e function, which is in this case a purely integral function (also called Persidskii function). Hence, Theorem 3 reduces to a test of robust diagonal stability of $\tilde{A}(\alpha)$. Additionally, using Theorem 3 with matrix coefficients $P_{k}=\mathbf{0}$, that is, with the Lur'e function reduced to a purely quadratic function, one has, from [10, Lemma 11], that the feasibility of (15)-(17) is equivalent to the fact that the $\mathscr{H}_{\infty}$ norm of the transfer function

$$
M(s, \alpha)=\frac{1}{2} Q_{g}(\alpha)^{1 / 2}(\Gamma-\Delta)\left(s \mathbf{I}-\frac{1}{2} \tilde{A}(\alpha)(\Gamma+\Delta)\right)^{-1} \tilde{A}(\alpha) Q_{g}(\alpha)^{-1 / 2}
$$

is less than one, $\forall \alpha \in \mathscr{U}$.

As a final remark, consider the case where the parameters of the sector $\mathscr{F}(\gamma, \delta)$ are not known. In this case, the conditions of Theorem 3 can also be used to optimize the sector 
parameters, which allows, for instance, to estimate the domain of stability of systems subject to saturation nonlinearities. Notice that amplitude saturating state variables can be represented by choosing $\Delta=\mathbf{I}$ and $\Gamma=\gamma \mathbf{I}$ in (4). Then, the solution of the optimization problem min $\gamma$ subject to (14)-(17) allows to obtain the best estimate of the domain of stability using sector (4) to represent the saturation and the conditions of Theorem 3 for stability. Other strategies to optimize the sector parameters can be found on [10, Section 6].

A convex solution to Problem 2 is given in the next theorem.

Theorem 4 Given diagonal matrices $\Gamma=\operatorname{diag}\left\{\gamma_{i}\right\}, \Delta=\operatorname{diag}\left\{\delta_{i}\right\}$, with $0<\gamma_{i} \leq 1 \leq \delta_{i}, i=$ $1, \ldots, n$, if there exist diagonal positive definite matrices $S \in \mathbb{R}^{n \times n}, R_{i} \in \mathbb{R}^{n \times n}, i=1, \ldots, N$, matrices $Z \in \mathbb{R}^{m \times n}$ and $\tilde{Z} \in \mathbb{R}^{m \times n}$ such that

$$
\begin{gathered}
\mathscr{M}_{i} \triangleq\left[\begin{array}{cc}
\mathscr{V}_{11} & \mathscr{V}_{12} \\
\star & \mathscr{V}_{22}
\end{array}\right]<0, i=1, \ldots, N \\
\mathscr{V}_{11} \triangleq S \tilde{A}_{i}^{\prime}+\tilde{Z}^{\prime} B_{i}^{\prime}+\tilde{A}_{i} S+B_{i} \tilde{Z}-R_{i} \\
\mathscr{V}_{12} \triangleq S \tilde{A}_{i}^{\prime}+\tilde{Z}^{\prime} B_{i}^{\prime}+A_{i} S+B_{i} Z+\frac{1}{2}(\Gamma+\Delta) R_{i} \\
\mathscr{V}_{22} \triangleq S A_{i}^{\prime}+Z^{\prime} B_{i}^{\prime}+A_{i} S+B_{i} Z-\Gamma \Delta R_{i}
\end{gathered}
$$

then the control gains

$$
K=Z S^{-1}, \tilde{K}=\tilde{Z} S^{-1}
$$

ensure that the closed-loop system (6) is GAS to $x=\mathbf{0}, \forall \alpha \in \mathscr{U}, \forall f \in \mathscr{F}(\gamma, \delta)$.

Proof: If Theorem 4 is feasible, one has that $\mathscr{M}(\alpha)=\sum_{i=1}^{N} \alpha_{i} \mathscr{M}_{i}<0, \forall \alpha \in \mathscr{U}$, with

$$
\begin{gathered}
\mathscr{M}(\alpha)=\left[\begin{array}{cc}
\mathscr{V}_{11}(\alpha) & \mathscr{V}_{12}(\alpha) \\
\star & \mathscr{V}_{22}(\alpha)
\end{array}\right] \\
\mathscr{V}_{11}(\alpha)=S \tilde{A}_{c l}(\alpha)^{\prime}+\tilde{A}_{c l}(\alpha) S-R(\alpha) \\
\mathscr{V}_{12}(\alpha)=S \tilde{A}_{c l}(\alpha)^{\prime}+A_{c l}(\alpha) S+\frac{1}{2}(\Gamma+\Delta) R(\alpha) \\
\mathscr{V}_{22}(\alpha)=S A_{c l}(\alpha)^{\prime}+A_{c l}(\alpha) S-\Gamma \Delta R(\alpha)
\end{gathered}
$$

with $A_{c l}(\alpha)$ and $\tilde{A}_{c l}(\alpha)$ given by (6). Pre and post-multiplying $\mathscr{M}(\alpha)$ by $\operatorname{diag}\left\{S^{-1}, S^{-1}\right\}$ and using the variable transformation $S^{-1}=P$, one gets (9), with $A(\alpha)=A_{c l}(\alpha)$ and $\tilde{A}(\alpha)=\tilde{A}_{c l}(\alpha)$ and with $P_{g}(\alpha)=P, W_{g}(\alpha)=P$ and $Q_{g}(\alpha)=S^{-1} R(\alpha) S^{-1}$. Thus, the feasibility of Theorem 4 ensures the existence of a Lur'e function

$$
v(x)=x^{\prime} P x+2 \sum_{i=1}^{n} p_{i i} \int_{0}^{x_{i}} f_{i}(\tau) d \tau
$$

which certifies the robust absolute stability of the closed-loop system.

It is worth of mention that the conditions of Theorem 4 are also valid for the case of timevarying uncertain parameters with unknown (even unbounded) rates of variation, lying in the 
unit simplex, since these conditions are based on a Lur'e function with a fixed matrix $P$. Observe also that decentralized control design can be directly addressed through Theorem 4 by using block-diagonal structure in matrices $Z$ and $\tilde{Z}$. As a final remark, notice that the results from Theorem 4 can also be applied to the case of purely linear state feedback stabilization (i.e. $u=$ $K x$ ), by setting to zero matrix $\tilde{Z}$, or to the case of purely nonlinear state feedback stabilization (i.e. $u=\tilde{K} f(x)$ ), by zeroing matrix $Z$.

\section{Examples}

Example 1 Consider system (1) with $A(\alpha)=\mathbf{0}, u=\mathbf{0}$ and with vertices

$$
\begin{aligned}
\tilde{A}_{1} & =\left[\begin{array}{cccc}
-1.94 & 0.25 & 0.63 & 0.38 \\
0.81 & -1.57 & 0.77 & 0.80 \\
0.60 & 0.33 & -1.49 & 0.69 \\
0.24 & 0.33 & 0.76 & -1.46
\end{array}\right] \\
\tilde{A}_{2} & =\left[\begin{array}{cccc}
-1.59 & 0.24 & 0.44 & 0.86 \\
0.22 & -1.27 & 0.76 & 0.59 \\
0.80 & 0.57 & -1.84 & 0.39 \\
0.42 & 0.52 & 0.96 & -2.16
\end{array}\right] \\
\tilde{A}_{3} & =\left[\begin{array}{cccc}
-2.03 & 0.85 & 0.19 & 0.72 \\
0.87 & -2.10 & 0.87 & 0.80 \\
0.23 & 0.79 & -1.94 & 0.95 \\
0.98 & 0.44 & 0.39 & -1.96
\end{array}\right]
\end{aligned}
$$

The objective of this example is to estimate the domain of robust stability when the system is subject to saturating state variables given by

$$
f_{(i)}\left(x_{i}\right)=\operatorname{sat}\left(x_{i}\right)= \begin{cases}\rho & \text { if } x_{i}>\rho \\ x_{i} & \text { if }-\rho \leq x_{i} \leq \rho \\ -\rho & \text { if } x_{i}<-\rho\end{cases}
$$

with $i=1, \ldots, 4$ and $\rho=1$. The sector (4) can be used to (locally) represent the saturation (25), by choosing the sector parameters as $\Delta=\mathbf{I}$ and $\Gamma=\gamma \mathbf{I}$. Then, to get estimates of the domain of robust stability for this nonlinear system, the problem $\gamma^{*} \triangleq \min \gamma$ subject to (14)-(17) is solved for given $g$ and $d$. Notice that the estimate of the domain of robust stability using this approach is given by

$$
\mathscr{S}=\left\{x \in \mathbb{R}^{4}:\left|x_{i}\right| \leq \frac{1}{\gamma^{*}}, i=1, \ldots, 4\right\}
$$

Table 1 summarizes the results. From Table 1, it is possible to observe that Theorem 3 solved using parameter-independent matrices (i.e. $g=0$ ) cannot provide any information on the estimate of the domain of robust stability for this system, even with the increase of $d$. On the other hand, the conditions of Theorem 3 with HPPD matrices yield estimates of the domain of robust stability that are progressively less conservative as one increases $g$ and $d$. Notice that for 
Table 1: Estimates of the domain of robust stability of system of Example 1, given by region $\mathscr{S}$, with $\gamma^{*} \triangleq \min \gamma$ s.t. (14)-(17) $\Delta=\mathbf{I}, \Gamma=\gamma \mathbf{I}, A_{i}=\mathbf{0}, i=1, \ldots, 3$, and $\tilde{A}_{i}$ given by (22)-(24).

\begin{tabular}{c|c|c|c}
\hline \hline$g$ & \multicolumn{3}{|c}{$\gamma^{*}$} \\
\cline { 2 - 4 } & $d=0$ & $d=1$ & $d=2$ \\
\hline 0 & \multicolumn{3}{|c}{ unfeasible } \\
\hline 1 & 0.72 & 0.62 & 0.57 \\
2 & 0.29 & 0.24 & 0.20 \\
3 & 0.20 & 0.16 & 0.14 \\
4 & 0.14 & 0.11 & 0.08 \\
5 & 0.09 & 0.07 & 0.06 \\
\hline \hline
\end{tabular}

a given $g$, the conservatism of the results are reduced by increasing $d$, which is based on the relaxation of the LMI constraints of the problem. Notice also that for a given $d$, the increase on $g$ allows to get better results due to the use of more scalar variables in the problem. Note the great improvement in terms of $\gamma^{*}$, reduced from 0.72 to 0.06 , with the increase of $g$ and $d$.

Example 2 As an example dealing with control design, consider system (1) with vertices given by

$$
\begin{aligned}
& A_{1}=\left[\begin{array}{ll}
0.30 & 0.19 \\
0.19 & 0.68
\end{array}\right], A_{2}=\left[\begin{array}{ll}
0.50 & 0.82 \\
0.90 & 0.64
\end{array}\right] \\
& \tilde{A}_{1}=\left[\begin{array}{ll}
0.30 & 0.15 \\
0.54 & 0.70
\end{array}\right], \tilde{A}_{2}=\left[\begin{array}{ll}
0.82 & 0.34 \\
0.66 & 0.29
\end{array}\right] \\
& B_{1}=\left[\begin{array}{ll}
0.38 & 0.85 \\
0.86 & 0.59
\end{array}\right], B_{2}=\left[\begin{array}{ll}
0.34 & 0.73 \\
0.53 & 0.31
\end{array}\right]
\end{aligned}
$$

The sector is defined as $\Gamma=10^{-\eta_{1}} \mathbf{I}$ and $\Delta=10^{\eta_{2}} \mathbf{I}$, where $\eta_{1}$ and $\eta_{2}$ can assume values on the natural numbers. The objective here is to stabilize this system for the maximum value of $\eta_{1}+\eta_{2}$, for all $\alpha \in \mathscr{U}$. As a first evaluation, one has that for the purely linear state feedback control law $u=K x$, Theorem 4 can ensure stability for $\Gamma=10^{-12} \mathbf{I}$ and $\Delta=10^{3} \mathbf{I}$, that is, for $\eta_{1}+\eta_{2}=15$. In the case of the purely nonlinear state feedback control law $u=\tilde{K} f(x)$, Theorem 4 ensures stability for $\Gamma=10^{-3} \mathbf{I}$ and $\Delta=10^{12} \mathbf{I}$, resulting in $\eta_{1}+\eta_{2}=15$. When both linear state vector and nonlinear state vector are used in the control law $u=K x+\tilde{K} f(x)$, Theorem 4 ensures closed-loop stability for $\Gamma=10^{-12} \mathbf{I}, \Delta=10^{12} \mathbf{I}$, yielding $\eta_{1}+\eta_{2}=24$, thus illustrating that the simultaneous use of linear and nonlinear feedback can enlarge the domains of stability of the closed-loop system. For $\Gamma=10^{-12} \mathbf{I}$ and $\Delta=10^{12} \mathbf{I}$, the stabilizing gains provided by Theorem 4 are given by

$$
K=\left[\begin{array}{cc}
8.86 & 0.74 \\
-6.05 & -5.11
\end{array}\right], \tilde{K}=\left[\begin{array}{cc}
8.41 & 1.58 \\
-5.68 & -5.31
\end{array}\right]
$$

and matrix $S$ is given by

$$
S=\left[\begin{array}{cc}
17846.43 & 0.00 \\
0.00 & 22091.81
\end{array}\right]
$$


being $P=S^{-1}$ the matrix of the Lur'e function (21) that ensures closed-loop robust absolute stability for any arbitrary value of time-derivative of the uncertain parameters $\alpha$.

\section{Conclusion}

This paper provides LMI conditions whose solution allows to construct homogeneous polynomially parameter-dependent Lur'e functions of general degree in the parameters. The existence of such functions ensures the robust absolute stability of polytopic systems perturbed by statedependent sector nonlinearities which lie in a general class of sectors. Given a degree for the Lur'e candidate function, progressively less conservative LMI relaxations that assess the robust absolute stability of the system are provided. Previous conditions in the literature for robust stability of linear systems and for absolute stability of precisely known nonlinear systems are recovered from the proposed conditions as special cases. An extension for robust control design applied to the class of nonlinear systems under investigation is also proposed, proving to be a useful convex tool to compute a stabilizing controller using only linear or nonlinear state feedback or using both linear and nonlinear state feedback terms in the control law.

\section{References}

[1] F. Albertini and D. D'Alessandro. Asymptotic stability of continuous-time systems with saturation nonlinearities. Systems \& Control Letters, 29(3):175-180, 1996.

[2] M. Arcak and P. Kokotovic. Feasibility conditions for circle criterion designs. Systems \& Control Letters, 42(5):405-412, April 2001.

[3] M. Arcak, M. Larsen, and P. Kokotovic. Circle and Popov criteria as tools for nonlinear feedback design. Automatica, 39(4):643-650, April 2003.

[4] B. R. Barmish. Necessary and sufficient conditions for quadratic stabilizability of an uncertain system. Journal of Optimization Theory and Applications, 46(4):399-408, August 1985.

[5] P.-A. Bliman. Absolute stability criteria with prescribed decay rate for finite-dimensional and delay systems. Automatica, 38(11):2015-2019, November 2002.

[6] P.-A. Bliman. An existence result for polynomial solutions of parameter-dependent LMIs. Systems \& Control Letters, 51(3-4):165-169, March 2004.

[7] P.-A. Bliman, R. C. L. F. Oliveira, V. F. Montagner, and P. L. D. Peres. Existence of homogeneous polynomial solutions for parameter-dependent linear matrix inequalities with 
parameters in the simplex. In Proceedings of the 45th IEEE Conference on Decision and Control, pages 1486-1491, San Diego, CA, December 2006.

[8] S. Boyd, L. El Ghaoui, E. Feron, and V. Balakrishnan. Linear Matrix Inequalities in System and Control Theory. SIAM Studies in Applied Mathematics, Philadelphia, PA, 1994.

[9] G. Chesi, A. Garulli, A. Tesi, and A. Vicino. Polynomially parameter-dependent Lyapunov functions for robust stability of polytopic systems: An LMI approach. IEEE Transactions on Automatic Control, 50(3):365-370, March 2005.

[10] M. C. de Oliveira, J. C. Geromel, and L. Hsu. A new absolute stability test for systems with state-dependent perturbations. International Journal of Robust and Nonlinear Control, 12(14):1209-1226, December 2002.

[11] P. Gahinet, P. Apkarian, and M. Chilali. Affine parameter-dependent Lyapunov functions and real parametric uncertainty. IEEE Transactions on Automatic Control, 41(3):436-442, March 1996.

[12] P. Gahinet, A. Nemirovskii, A. J. Laub, and M. Chilali. LMI Control Toolbox User's Guide. The Math Works Inc., Natick, MA, 1995.

[13] J. C. Geromel, M. C. de Oliveira, and L. Hsu. LMI characterization of structural and robust stability. Linear Algebra and Its Applications, 285(1-3):69-80, December 1998.

[14] G. H. Hardy, J. E. Littlewood, and G. Pólya. Inequalities. Cambridge University Press, Cambridge, UK, 2 edition, 1952.

[15] D. Henrion, D. Arzelier, D. Peaucelle, and J. B. Lasserre. On parameter-dependent Lyapunov functions for robust stability of linear systems. In Proceedings of the 43rd IEEE Conference on Decision and Control, pages 887-892, Paradise Island, Bahamas, December 2004.

[16] E. Kaszkurewicz and A. Bhaya. Robust stability and diagonal Liapunov functions. SIAM Journal on Matrix Analysis and Applications, 14:508-520, 1993.

[17] E. Kaszkurewicz and A. Bhaya. Matrix Diagonal Stability in Systems and Computation. Birkhäuser, Boston, MA, 1999.

[18] H. K. Khalil. Nonlinear Systems. Prentice Hall, Upper Saddle River, NJ, 1996.

[19] R. C. L. F. Oliveira and P. L. D. Peres. LMI conditions for the existence of polynomially parameter-dependent Lyapunov functions assuring robust stability. In Proceedings of the 
44th IEEE Conference on Decision and Control - European Control Conference ECC 2005, pages 1660-1665, Seville, Spain, December 2005.

[20] R. C. L. F. Oliveira and P. L. D. Peres. Stability of polytopes of matrices via affine parameter-dependent Lyapunov functions: Asymptotically exact LMI conditions. Linear Algebra and Its Applications, 405:209-228, August 2005.

[21] R. C. L. F. Oliveira and P. L. D. Peres. LMI conditions for robust stability analysis based on polynomially parameter-dependent Lyapunov functions. Systems \& Control Letters, 55(1):52-61, January 2006.

[22] D. Peaucelle, D. Arzelier, O. Bachelier, and J. Bernussou. A new robust $\mathscr{D}$-stability condition for real convex polytopic uncertainty. Systems \& Control Letters, 40(1):21-30, May 2000 .

[23] S. K. Persidskii. Problem of absolute stability. Automation and Remote Control, 12:1889$1895,1969$.

[24] V.-M. Popov. On the absolute stability of nonlinear controlled systems. Avtomatika $i$ telemekhanika, 8:961-970, 1961.

[25] D. C. W. Ramos and P. L. D. Peres. An LMI condition for the robust stability of uncertain continuous-time linear systems. IEEE Transactions on Automatic Control, 47(4):675-678, April 2002.

[26] C. W. Scherer. Higher-order relaxations for robust LMI problems with verifications for exactness. In Proceedings of the 42nd IEEE Conference on Decision and Control, pages 4652-4657, Maui, HI, USA, December 2003.

[27] C. W. Scherer. Relaxations for robust linear matrix inequality problems with verifications for exactness. SIAM Journal on Matrix Analysis and Applications, 27(2):365-395, 2005.

[28] C. W. Scherer and C. W. J. Hol. Matrix sum-of-squares relaxations for robust semi-definite programs. Mathematical Programming Series B, 107(1-2):189-211, June 2006.

[29] J. F. Sturm. Using SeDuMi 1.02, a MATLAB toolbox for optimization over symmetric cones. Optimization Methods and Software, 11-12:625-653, 1999. URL: http://sedumi.mcmaster.ca/.

[30] J. C. Willems. Least squares stationary optimal control and algebraic Riccati equation. IEEE Transactions on Automatic Control, AC-16(6):621-634, December 1971. 